\title{
Principles and application of velocimetry-based planar pressure imaging in compressible flows with shocks
}

\author{
B. W. van Oudheusden
}

Received: 27 October 2007 / Revised: 17 July 2008 / Accepted: 20 July 2008 / Published online: 6 August 2008

(C) The Author(s) 2008. This article is published with open access at Springerlink.com

\begin{abstract}
The paper addresses the computation of pressure fields from velocimetry data, such as provided by Particle Image Velocimetry (PIV), with specific attention to its application in compressible flows with shocks. An essential extension with respect to incompressible flow is that in view of the variable density occurring in compressible flow, the velocimetry data has to be supplemented with additional relations, derived from the flow governing equations. Secondly, compressible flows display specific flow features, notably shocks but also thin shear layers, that pose particular difficulties for the flow velocity measurement itself, as well as for the subsequent determination of the pressure field. The present communication addresses the basic principles of the pressure-field extraction method, as well as its feasibility of application under realistic experimental conditions.
\end{abstract}

\section{Introduction}

Particle Image Velocimetry (PIV) is currently employed as a reliable quantitative flow diagnostic tool in a large variety of aeronautical regimes, ranging from low speed (incompressible) to highly compressible conditions (supersonic and hypersonic flows). As its basic characteristic, the PIV technique provides an instantaneous characterisation of the velocity field that provides direct experimental evidence of

B. W. van Oudheusden ( $\square)$

Department of Aerospace Engineering,

Delft University of Technology,

Kluyverweg 1, 2629 HS Delft, The Netherlands

e-mail: b.w.vanoudheusden@tudelft.nl the flow phenomena under investigation, as well as offering a detailed and high-quality validation data base for CFD simulations. In addition to the velocity field information directly produced by the PIV technique, a post-processing of the flow field data allows to extract, under certain restrictions, also the pressure field. The primary motivation for such an approach is to obtain estimates of the thermodynamic properties (pressure, but also temperature and density) once the flow velocity information is available from measurement. This is considered a fluid dynamic topic of interest in itself, that may further assist interpretation of flow phenomena observations by means of velocimetry (such as PIV or LDA), but also has direct technical relevance in relation to distributed pressure loads and total resulting forces on objects (hence, the particular focus on the pressure). Indeed, these properties can also be obtained by other means (balances, pressure holes or PSP), but the velocimetry-based approach offers the perspective of: (1) permitting a direct correlation between loads and the flow features responsible, (2) simplifying experimental model manufacture (no balances, pressure tapping required), (3) offering the potential of pressure 'measurement' with more flexibility and higher spatial resolution than with pressure tap systems.

Under the particular limitation of isentropic flow, a direct algebraic relation applies between the pressure and the velocity (similar to Bernoulli's relation for incompressible flow):

$\frac{p}{p_{\infty}}=\left(\frac{T}{T_{\infty}}\right)^{\frac{\gamma}{\gamma-1}}=\left(1+\frac{\gamma-1}{2} M_{\infty}^{2}\left(1-\frac{V^{2}}{V_{\infty}^{2}}\right)\right)^{\frac{\gamma}{\gamma-1}}$

where $V$ is the velocity magnitude and with $V_{\infty}$ and $M_{\infty}$ the velocity and Mach number of the free stream (the ratio of specific heats is $\gamma=1.4$ for air). Although in many aeronautical applications the above assumptions of isentropic flow may be valid for a large domain of the flow, in which 
case the computation of the pressure becomes quite trivial, clearly the isentropic flow assumption is violated in regions affected by shock waves and in viscous shear layer regions (boundary layers, wakes). Therefore, the explicit topic of the present investigation is to develop a procedure of more general applicability.

\subsection{Relation to previous work}

In more general terms, the pressure determination principle relies on the fact that through the momentum equation, the pressure gradient can be determined from the velocity field information, which delivers the pressure field upon subsequent spatial integration (see, e.g. Unal et al. 1997; Baur and Köngeter 1999; Gurka et al. 1999). In the case of twodimensional flow, the required velocity data can be measured with planar PIV. For an unsteady but statistically stationary flow, the mean pressure can be related to the velocity field statistics in a similar way through a Reynolds-averaging approach (Van Oudheusden et al. 2007). The aspect of instantaneous pressure determination will not be considered here, in view of the complexity of obtaining reliable flow acceleration data under high-speed conditions.

The extension of this pressure-integration procedure to the compressible flow regime has been considered recently (Van Oudheusden et al. 2007; Souverein et al. 2007; Van Oudheusden and Souverein 2007). In view of the variable density occurring in compressible flow, the velocimetry data and momentum equation need to be supplemented with additional relations, which are derived from the flow governing equations. Secondly, compressible flows display specific flow features, notably shocks but also thin shear layers, that pose particular difficulties in the velocity measurement itself, as well as in the subsequent pressuregradient integration. In the previous studies referred to above, a convenient solution approach to remove the density dependence was adopted, using the gas law and the adiabatic flow condition. The method was subsequently applied in a PIV-based loads determination of an airfoil in supersonic flow, for which purpose the pressure was computed only on a contour surrounding the airfoil. A special shock treatment was applied by first identifying and characterizing the shocks (position and inclination, hence, deducing shock strength) from the velocity field, by means of an interactive graphical procedure. Subsequently, the shock regions were masked and the pressure integration along the contour was propagated over the shocks using theoretical shock relations (Souverein et al. 2007).

With respect to this earlier work, the aims of the present investigation are to extend and generalize the procedure to an area integration method, in order to obtain spatial pressure fields, as well as to investigate the feasibility of applying the spatial integration procedure across shock regions, which if successful would eliminate the need for the explicit shock treatment.

The basic concepts of the pressure-field extraction method are further outlined in the next section, illustrated in relation to a schematic compressible flow field that may be considered representative of a typical aeronautical highspeed flow application. The purpose of this section is to discuss, at a more qualitative and conceptual level first, the different approaches that need to be taken to obtain pressure from velocity data under typical compressible flow conditions, depending on the flow regime. As a conclusion from this discussion, a number of sub-objectives are identified, in terms of the pressure-gradient formulation and the numerical integration strategy. The description of the pressure determination procedure and its specific implementation are discussed in Sect. 2; the 'predicted performance' of the proposed strategy is analysed in Sect. 3 , based on a theoretical analysis, while the particular behaviour of the integration across a shock region is addressed in Sect. 4, using theoretical analysis, a simulated experiment and experimental shock test data. Finally, Sect. 5 contains applications to two experimental data sets: the supersonic flow around a biconvex airfoil and a shock wave boundary layer interaction.

\subsection{Concept of the pressure determination approach}

The aim of the present investigation is to develop and assess a generalized procedure for computing the thermodynamic properties (pressure in particular) for a compressible flow field, where the velocity is known from measurement. To support the following discussion, an example compressible flow field is given in the schematic of Fig. 1, representing the supersonic flow around a bluntnosed object with a truncated base. It displays a number of

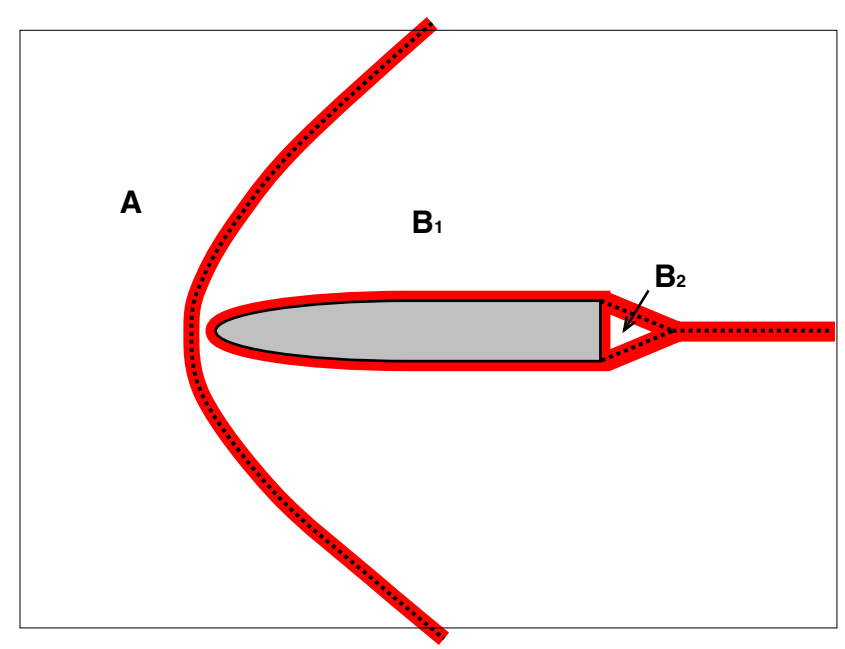

Fig. 1 Schematic representation of flow field regimes in relation to the pressure computation procedure 
typical high-speed flow features, such as a detached, curved bow shock in front of the object, thin boundary layers along the surfaces and a separated wake flow behind it. In order to derive the pressure (as well as density and temperature) from the local velocity obtained from measurement, a suitable theoretical flow model needs to be applied when post-processing the available velocity data. In this respect, different flow regions are distinguished, as indicated in the figure.

In the region marked "A", the flow can be assumed inviscid and irrotational, hence, the flow can be considered isentropic and the isentropic relations, like Eq. (1), can be applied to compute the local pressure directly from the local velocity. In the particular example, moreover, this region upstream of the bow shock would be even a uniform flow region.

Secondly, in the regions marked " $\mathrm{B}$ " $\left(\mathrm{B}_{1}\right.$ and $\mathrm{B}_{2}$ in the current example) the flow is considered inviscid as well, but it can be rotational. This implies that although the flow in these regions has been affected by viscous processes (shear layers and/or shocks) the viscous stresses themselves are no longer significant. As a result, the flow entropy remains constant along streamlines, but it is not constant in the entire flow region and likely it is also different from the free stream value. Pressure determination by means of applying the isentropic relations along streamlines is not a very convenient pressure-determination strategy for such regions. Not only would this require identification of the streamlines, but also upstream boundary conditions need to be prescribed for each streamline separately, which may be especially problematic for separated flow regions of type " $\mathrm{B}_{2}$ ". Instead, a more flexible approach is to invoke the momentum equation in differential form to compute the local pressure gradient, and then determine the pressure from subsequent spatial integration. This approach requires only a limited region (or even a single point) of prescribed pressure as boundary condition. Note that regions of both type "A" and 'B" are governed by the inviscid flow equations alike (the Euler equations), which means that also the region of type "A" or parts of it may be included in the integration domain.

Finally, the regions indicated in red in Fig. 1 are those regions where the flow is essentially viscous and where in addition to that the flow velocity measurement is likely to be severely compromised due to lack of spatial resolution and/or tracer particle inertia effects. Typically this corresponds to regions where shocks and shear layers occur in the flow field. These regions pose particular challenges to the pressure determination: whereas viscous terms can be significant in the momentum balance, these cannot be properly resolved. Nevertheless, the treatment of these regions can form an essential element in the pressure determination procedure as a whole, for example where they provide the link between different regions, like the bow shock between regions " $A$ " and " $B_{1}$ ", or the shear layer between " $\mathrm{B}_{1}$ " and " $\mathrm{B}_{2}$ ". A possible approach is to exclude these regions from the actual pressure-integration procedure and treat them explicitly, as suggested previously. This involves identification of these regions, their location, extent and nature, and the subsequent propagation of the pressure-integration results from the reliable regions across them, by means of appropriate theoretical flow relations. Evidently, the automation of the pressure-computation procedure would be much facilitated if this explicit treatment of the viscous regions can be avoided, and the integration procedure could be applied across the viscous regions indiscriminately instead. It may be obvious that applying a theoretical flow model (like that of inviscid flow) in regions where this is not appropriate, will not provide correct pressure results, in particular when the velocity measurement in this regions is affected by serious error as well. Hence, such an approach can only be justified to the extent to which it is capable of correctly propagating flow information (pressure, density, temperature) across these regions, to serve as proper boundary or input conditions for the subsequent regions where the flow model is again correct. A particular objective of the present investigation therefore is to assess if such an approach is feasible, within acceptable accuracy, and whether this approach introduces systematic bias errors and if so, assess the nature and extent of these bias errors.

In summary, a flexible pressure-determination procedure for compressible flows is pursued and in view of the preceding considerations, the following specific objectives are identified as elements in its development and assessment:

1. to define a convenient pressure-gradient formulation that permits pressure computation through an integration approach that takes variable density into account, preferably with an explicit formulation so that the pressure can be determined in a non-iterative process;

2. to implement a flexible integration scheme with which the pressure gradient can be integrated on an arbitrary domain and with arbitrary initial value regions;

3 . to investigate the feasibility to apply the pressuregradient strategy across shock and shear layer regions, as an alternative to their explicit treatment.

\section{Description of the pressure determination procedure}

The computation of pressure from the velocity field basically relies on invoking the momentum equation (see, e.g. Unal et al. 1997), which for incompressible flow allows the pressure gradient to be related uniquely to velocity field variables. The pressure itself is subsequently obtained from a spatial integration of the pressure 
gradient. The momentum equation is also employed as the fundamental relation for obtaining the pressure gradient in the case of compressible flow, but additional relations must be included to account for the variable density. As stated, a further objective of the present work, with practical benefit, is to provide a formulation that allows the gradient of the pressure (or some related function) to be expressed exclusively in terms of properties that can be obtained directly from the velocity field data, i.e. formulating the problem in symbolic form as:

$\nabla f(p)=g(\mathbf{V})$

Such an approach has the convenient characteristic that it allows the computation of the pressure field in a noniterative way (no updating steps to account for variable density are required, hence, no potential problem of numerical stability of the procedure), while in addition the same numerical procedures for gradient-integration can be used as for incompressible flow (see next section).

In the following discussion a number of simplifying assumptions will be adopted. Firstly, the flow model used to compute the pressure gradient will assume steady flow (alternatively, mean pressures may be computed using the Reynolds averaging concept). Also, the viscous terms in the momentum equation will be discarded, in view of the consideration that these either have a negligible influence (for example when considering the pressure gradient normal to a viscous shear layer region) or that they cannot be properly resolved from the measured velocity field. Note that this assumption does not imply that the procedure is only valid in the case of inviscid flow, but only that the contribution of the viscous stresses is not taken explicitly into account when computing the pressure gradient. Under these assumptions, the momentum equation reads:

$\nabla p=-\nabla(\rho \mathbf{V} \mathbf{V})=-\rho(\mathbf{V} \cdot \nabla) \mathbf{V}$

The two alternative expressions reflect the conservative and non-conservative formulation of the momentum equation, respectively. In theory these formulations are perfectly equivalent as a consequence of mass conservation, which the real flow will evidently observe. However, pressure-gradient integration schemes using either of the two proposed alternative formulations may not necessarily produce the same results when mass conservation is not explicitly imposed and when the conditions are such that the computed results do not agree with continuity. The latter is potentially the case when this essentially inviscid model is applied to a measured velocity field region that contains departure from an inviscid flow solution. Note that at this phase of the discussion we only make the observation that there can be a different outcome from using the two schemes, but no conclusion can yet be drawn on its consequences, more particular if one strategy will work better than the other. For this reason the possible difference in performance of the two alternative formulations will be one of the specific points of interest in the following analysis (see also Sects. 3.2 and 4.1).

\subsection{Explicit pressure-gradient formulations for compressible flow}

As in the case of compressible flow the density appears as an extra unknown in the momentum equation, additional flow equations are invoked for the pressure determination. With the velocity field provided from measurement, the complete set of governing equations makes the pressure computation overspecified. Hence, one may attempt to optimize pressure computation for example in the least squares sense, or alternatively opt to use only a sufficient subset of the equations. In the latter case, one should be aware that the final results may potentially not satisfy the physical conservation principles expressed by the discarded equations.

A convenient approach for incorporating the variable density in the momentum equation was adopted that combines the gas law and the adiabatic flow assumption, as these are both expressed by algebraic relations. The gas law is used to replace the density by relating it to pressure and temperature, while the assumption of constant total temperature is employed to express temperature in terms of the velocity magnitude $V$ :

$\rho=\frac{p}{R T} ; \quad \frac{T}{T_{\infty}}=1+\frac{\gamma-1}{2} M_{\infty}^{2}\left(1-\frac{V^{2}}{V_{\infty}^{2}}\right)$

The adiabatic flow assumption applies in inviscid flow regions and over shocks, but also it is reasonable for viscous regions, in the case of steady flow without significant heat transfer, as expressed by the Crocco-Busemann relation (White 1991). Note that with this relation the temperature field is directly linked to the measured velocity, independent of the pressure integration strategy. Introducing these expressions in the momentum equation (3) allows, with some manipulation, to formulate the pressure-gradient components exclusively in terms of the velocity field, with respect to a Cartesian coordinate frame: with the nonconservative formulation of the momentum equation:

$\frac{1}{p} \frac{\partial p}{\partial x_{i}}=\frac{\partial \ln \left(p / p_{\infty}\right)}{\partial x_{i}}=-\frac{1}{R T} u_{j} \frac{\partial u_{i}}{\partial x_{j}}$

with the conservative formulation of the momentum equation:

$\left(\delta_{i j}+\frac{u_{i} u_{j}}{R T}\right) \frac{\partial \ln \left(p / p_{\infty}\right)}{\partial x_{j}}=-\frac{1}{R T}\left(\frac{\partial u_{i} u_{j}}{\partial x_{j}}-\frac{u_{i} u_{j}}{T} \frac{\partial T}{\partial x_{j}}\right)$

In the case of the conservative formulation, see Eq. (6), the pressure-gradient components for a $2 \mathrm{D}$ flow are obtained from solving a $2 \times 2$ set of equations. The 
outcome in both cases is that the pressure-integration procedure has been cast in essentially similar functional form as for the incompressible case, cf. Eq. (2), allowing the same numerical integration routines to be applied for a non-iterative computation of the pressure.

In the case of a turbulent flow the contribution of the turbulent stresses, provided they are properly resolved by the measurement, may be included in the pressure-gradient formulation, starting from the Reynolds-averaged form of the momentum equation (3), similar as in the case for incompressible flow (Van Oudheusden et al. 2007). Neglecting the effect of density fluctuations (for moderate Mach numbers), the pressure gradient then follows from:

$\left(\delta_{i j}+\frac{\overline{u_{i}^{\prime} u_{j}^{\prime}}}{R T}\right) \frac{\partial \ln \left(p / p_{\infty}\right)}{\partial x_{j}}=-\frac{1}{R T}\left(u_{j} \frac{\partial u_{i}}{\partial x_{j}}+\frac{\partial \overline{u_{i}^{\prime} u_{j}^{\prime}}}{\partial x_{j}}-\frac{\overline{u_{i}^{\prime} u_{j}^{\prime}}}{T} \frac{\partial T}{\partial x_{j}}\right)$

(non-conservative)

$$
\begin{aligned}
& \left(\delta_{i j}+\frac{\overline{u_{i} u_{j}}}{R T}\right) \frac{\partial \ln \left(p / p_{\infty}\right)}{\partial x_{j}}=-\frac{1}{R T}\left(\frac{\partial \overline{u_{i} u_{j}}}{\partial x_{j}}-\frac{\overline{u_{i} u_{j}}}{T} \frac{\partial \mathrm{T}}{\partial x_{j}}\right) \\
& \quad \text { (conservative) }
\end{aligned}
$$

where: $\overline{u_{i} u_{j}}=u_{i} u_{j}+\overline{u_{i}^{\prime} u_{j}^{\prime}}$.

\subsection{Numerical gradient-integration strategy}

For computing the pressure from the velocity data as outlined above, the pressure gradient must be spatially integrated, with proper boundary conditions imposed. Basically two strategies have been proposed in literature for this kind of problems, that is either some sort of spatialmarching scheme to integrate the pressure gradient directly (see, e.g. Baur and Köngeter 1999; Liu and Katz 2006) or through solving the Poisson equation for the pressure (e.g. Gurka et al. 1999; Hosokawa et al. 2003; Fujisawa et al. 2005). Pressure boundary conditions may be prescribed in undisturbed flow regions, or through the direct use of isentropic flow relations, see Eq. (1), in those regions where they are appropriate.

In the present investigation a generalized routine for the numerical integration of the pressure-gradient was implemented, based on a field-erosion principle in combination with an averaged integration from neighbouring points, similar to the scheme used in Baur and Köngeter (1999). The field is divided in different regions: prescribed values, masked regions and integration regions. The integration proceeds by progressively extending the integration front from the prescribed-value region outwards. The progress of the integration is controlled by a parameter (Nmin) which is the prescribed minimum number of available neighbours required for integration to proceed. The effect of the value of Nmin on the progression and shape of the integration front is illustrated in Fig. 2, for an initially square region of initial values: for Nmin $=1$ it results in a rectangular expansion of the integration front, for Nmin $=2$ the front is bevelled in the diagonal direction, while for $\mathrm{Nmin}=3$ the expansion is more omni-directional (spherical) in shape. The overall numerical scheme as implemented in the present investigation is second-order accurate in space, with gradients computed with central differences and applying the trapezoid rule for integration. As the method should hence return exact results when the underlying pressure field is quadratic in space, so with velocity linear in space, this implies that the numerical truncation error is dominated by the velocity second-order spatial derivatives.

\subsection{Outline of the pressure-determination algorithm}

In summary, assuming that in the flow region of interest the velocity field has been obtained from measurement, the actual pressure-determination procedure proceeds as follows:
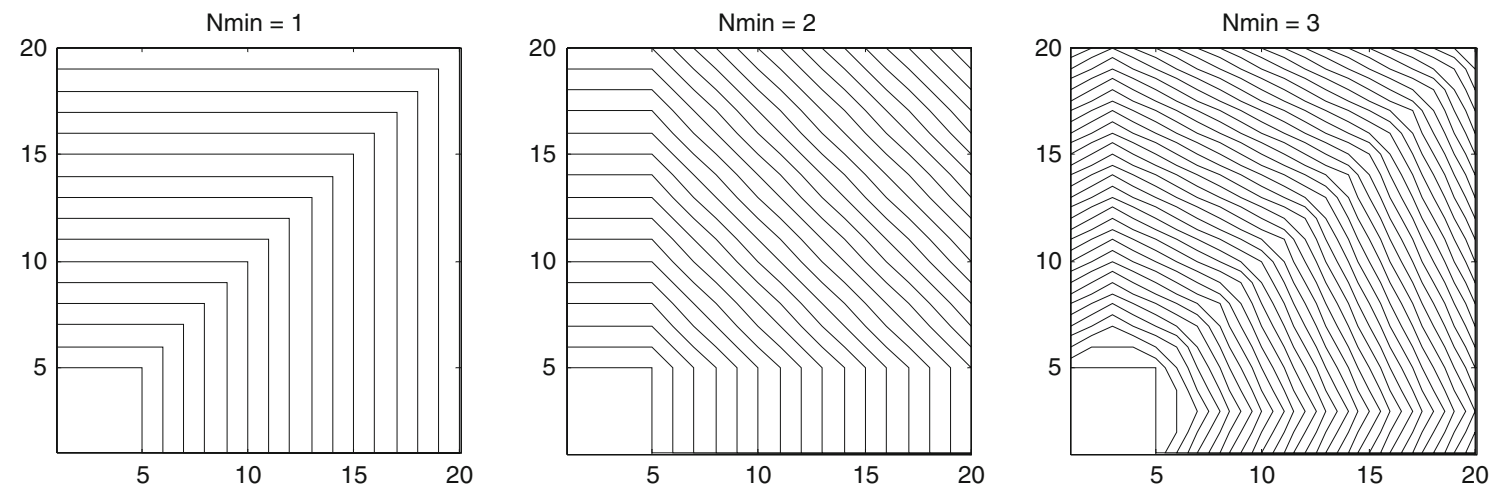

Fig. 2 Spatial integration strategy using an erosion procedure on a 20 $\times 20$ grid, starting from a $5 \times 5$ initial region in the lower left corner; the lines indicate the erosion front after each subsequent integration step. The erosion progress is controlled by the parameter Nmin, which is the minimum number of established neighbours required for subsequent integration 
- Step 1. The different regions in the measurement domain are identified: (1) an initial region where pressure will be prescribed, (2) regions where the pressure will be determined through the integration procedure and (3) masked regions which should be excluded from the spatial integration.

- Step 2: Using the adiabatic condition, Eq. (4b), the temperature field is computed.

- Step 3: The pressure value in the initial region is initialized, either by imposing the free-stream value or by invoking the isentropic relation, Eq. (1).

- Step 4: The momentum equation, either Eq. (5) or (6), or Eq. (7) or (8) when turbulence terms are included, is used to compute the pressure gradient in every point (or, actually the gradient of the logarithm of the pressure ratio).

- Step 5: The space-marching integration is applied to fill in the pressure information in the integration regions, starting from the initial domain-see Sect. 2.2.

- Step 6: As a post-processing step, the density may be computed from temperature and pressure, through the equation of state, Eq. (4a).

\section{Theoretical performance assessment of the pressure- determination procedure}

With the local pressure, temperature and density derived from the velocity field by the procedure outlined in the previous section, a full thermodynamic characterisation of the flow is obtained. In this respect it is important to appreciate the hybrid nature of the resulting data set, with one part of the information obtained from direct measurement (the velocity field) and another part (thermodynamic quantities) by inference, through invoking a theoretical flow model-where moreover, only a selection of the governing equations is involved. In the present approach we use an inviscid (not necessarily isentropic) flow model, and apply the momentum equation, energy equation (in the form of the adiabatic relation of constant total temperature) and the equation of state. The correctness of the 'measured flow' (velocity and thermodynamic quantities together) evidently depends on the correctness of the velocity measurement as well as on the appropriateness of the theoretical flow model applied. Moreover, systematic errors in velocity measurement and/or inappropriate flow modelling may potentially result in physical inconsistencies in the data set, in the sense that the 'measured flow' does not satisfy all the fundamental conservation principles of the true physical flow. For example, in the present procedure the conservation equation is not used, so mass conservation is not explicitly imposed. Therefore, an incorrect estimate of the density may result in that mass conservation is not obeyed by the 'measured flow' even when the velocity would have been measured correctly. This also indicates that the conservative and nonconservative formulations of the momentum equation may not necessarily produce the same results under all circumstances, which is the reason that they are considered as alternative approaches in the present investigation.

With reference to the flow schematic illustrated in Fig. 1, the flow regions of type ' $A$ ' and ' $B$ ' are expected to be handled in a predominantly correct way by the present procedure (provided that basic assumptions, such as 2D flow, apply), as velocity measurement is not affected by significant inaccuracies under these conditions and the assumption of inviscid flow is applicable there. The latter implies that the resulting data set is likely to be close to the 'true physical flow' and consistent with physical principles, even those not explicitly imposed in the determination of the thermodynamic properties. In particular, the results for the computed pressure and density are likely to agree well with continuity, and as a consequence the conservative and non-conservative formulations of the momentum equation will produce essentially similar results.

The situation is evidently more critical for the 'red regions' in Fig. 1 (corresponding notably to the surroundings of shock waves and shear layers), where the measurement will have a higher degree of uncertainty and where the theoretical flow model is incomplete. However, as indicated in the introduction, the objective of applying the pressure-integration procedure on these flow regions is not so much to produce correct pressure results inside the regions themselves, but rather to propagate flow information (pressure, density, temperature) across these regions, to serve as input conditions for the subsequent regions where the flow model is again correct.

\subsection{Accuracy considerations and velocity error propagation}

An aspect of evident relevance to the proposed procedure to compute pressure from the velocity data, is the extent to which the pressure outcome is influenced by inaccuracies in the velocity field data and in the pressure-determination procedure. Errors of a systematic nature can arise as a result of physical modelling deficiencies, and will be addressed in more detail in subsequent sections. Errors (uncertainties) of a more random nature, on the other hand, arise in the form of numerical integration errors, as well as due to the uncertainty in the underlying velocity information itself (measurement error, such as from PIV crosscorrelation uncertainty, etc.). The pressure computation error within the inviscid flow regions (type A and B) will be mostly determined by the latter two effects, while the physical modelling deficiencies may affect the way in 
which pressure information is propagated across the viscous flow regions. The objective of the present analytical assessment of the procedure's performance is twofold in this respect: (1) to assess how the accuracy of the pressure determination is related to uncertainties in the velocity information of a random nature and (2) to investigate the potential introduction of systematic errors when propagating flow information across shock wave and shear layer regions when the inviscid flow model is used.

As a first estimate of how the velocity error propagates into the pressure error, the direct relation between pressure and velocity for isentropic flow is considered, see Eq. (1), which allows assessment of how small variations of the velocity around its mean value affect the computed pressure in this case:

$\frac{\mathrm{d} p}{p}=\frac{\gamma}{\gamma-1} \cdot \frac{\mathrm{d} T}{T}=-\gamma M^{2} \frac{\mathrm{d} V}{V}$

or, alternatively, in terms of the pressure coefficient:

$\mathrm{d} C_{p}=\frac{\mathrm{d} p}{\frac{1}{2} \rho V^{2}}=\frac{\mathrm{d} p}{\frac{1}{2} \gamma p M^{2}}=-2 \frac{\mathrm{d} V}{V}$

These expressions reveal the error in $C_{p}$ to be equal to twice the relative error in the absolute velocity. Carefully conducted PIV experiments are known to deliver (timeaverage) velocity data with uncertainty well below $1 \%$, hence, pressure uncertainty can be kept within a similar limit as well. These results are based on a point-to-point computation of the pressure from the isentropic relation. The extent to which an integration procedure applied to the pressure gradient is expected to affect the pressure error, either accumulative or diminutive, is difficult to assess analytically. Simulated pressure-gradient integration tests show, however, that the integration procedure tends to smoothen the effect of purely random errors (noise).

\subsection{Thermodynamic consequences}

of the pressure-integration strategy

As stated previously, the choice of the inviscid flow model with which the pressure computation is performed can have an impact on the results, notably in the way in which pressure information is propagated across regions where the flow model is not appropriate (shocks and shear layers). A first analysis of the predicted behaviour of the proposed pressure-determination schemes and its consequence for the derived thermodynamic properties is carried out in this section, by investigating how the entropy field associated with the thermodynamic results that are returned from the integration, depends on the velocity information and how this may be affected by the characteristics of the particular pressure-integration scheme. By relating the entropy to local properties of the flow field in an analytical way, this approach enables to identify general characteristic features or systematic errors in the pressure computation, irrespective of how measurement uncertainty of the velocity and numerical truncation errors in the computation of spatial derivatives and the subsequent integration procedure may affect the integration results.

The first step in this analysis is to invoke Crocco's theorem (Anderson 2003), to relate the entropy gradient to the pressure, enthalpy and velocity:

$T \nabla s=\nabla h-\frac{\nabla p}{\rho}=\nabla H-\frac{\nabla p}{\rho}-(\mathbf{V} \cdot \nabla) \mathbf{V}-\mathbf{V} \times \boldsymbol{\omega}$

where $\boldsymbol{\omega}=\nabla \times \mathbf{V}$ is the vorticity. Note that the above relation has been derived from basic thermodynamic principles, plus the definition of the total enthalpy $\left(\nabla H=\nabla h+\nabla \frac{1}{2} \mathbf{V} \cdot \mathbf{V}\right)$ and the vectorial identity $\nabla\left(\frac{1}{2} \mathbf{V} \cdot \mathbf{V}\right)=(\mathbf{V} \cdot \nabla) \mathbf{V}+\mathbf{V} \times \boldsymbol{\omega}$ and, hence, still has general validity, irrespective of the flow model used for the post-processing. It may need to be stressed here again that this analysis intends to characterize the 'measured flow', meaning that in the above expressions the kinematic properties (velocity and vorticity) are the measured ones and the thermodynamic properties (pressure, temperature, density, entropy) are the inferred values from applying the theoretical flow model, so not the actual values of the true flow. Also, further manipulation should employ only the additional relations in the form they are actually used in the alternative pressure-integration schemes. In view of the adiabatic assumption, Eq. (4b), the total enthalpy gradient is zero, so $\nabla H=0$ for both schemes. Further, depending on the specific formulations of the momentum equation, conservative or non-conservative, see Eq. (3), the two alternative pressure-gradient schemes correspond to the following entropy gradient expressions:

$T \nabla s=-\mathbf{V} \times \boldsymbol{\omega} \quad$ (with the non-conservative formulation)

$T \nabla s=-\frac{\nabla \cdot(\rho \mathbf{V})}{\rho} \mathbf{V}-\mathbf{V} \times \boldsymbol{\omega}$

(with the conservative formulation)

Note that the above equations are not additional relations that are used in the actual pressure computation, but they are derived here to assess how the 'measured flow' is predicted to behave in terms of entropy and to characterize the impact of the integration scheme.

Both expressions contain a common term, which constitutes an entropy gradient component in the direction normal to the flow, which becomes zero for irrotational flow. The result for the conservative scheme contains an additional term which introduces an entropy gradient component in flow direction, and which is proportional to 
the extent to which the measured flow departs from mass conservation. As argued previously, the latter effect will be negligible in well-resolved inviscid flow regions. Also in shear layers the additional term is seen to be essentially small, as the velocity component in the dominant gradient direction (perpendicular to the shear layer) is small. Consequently, in inviscid flow regions as well as in shear layers both schemes will work essentially similar: the entropy (hence, total pressure) remains constant along a streamline and is moreover, constant on different streamlines in a domain where the flow is irrotational.

The situation is different for a shock wave. There, strong gradients in flow properties occur normal to the shock, hence in flow direction, which makes the additional term of possible significance. Therefore the two schemes are likely to produce different results under these conditions. Moreover, when using the non-conservative integration scheme across a normal or straight shock wave, in which case no rotation is produced in the velocity field, according to Eq. (12) the entropy (and total pressure) remains constant, hence, the pressure returned after the shock wave will be equal to the isentropic pressure, which is wrong.

In summary, the two pressure-integration schemes proposed on the basis of an inviscid flow model (Euler equations) are expected to perform well and provide similar results for essentially inviscid flow regions and across shear layers. Different behaviour is expected for the integration across a shock, with particular finding that the nonconservative scheme is not capable of reproducing total pressure loss over the shock. The latter observations motivate to further investigate the performance of the pressure-integration scheme across a shock wave region in more in detail in the next section.

\section{Assessment of pressure-gradient integration across a shock wave region}

Shock waves are regions specific to compressible flows that require special attention in the pressure determination, both from the fundamental fluid-dynamics perspective as well as in relation to the limitations of the PIV measurement technique. Shocks represent near-discontinuous features of the physical flow field, extending over a thickness comparable to the mean free molecular path, a scale for which there is evidently no diagnostic perspective. Also, viscous normal stresses essentially contribute to the momentum budget inside the shock, which means that neglecting these is likely to affect the result of the pressure integration result when this is applied unmodified over the shock region. Moreover, the velocity field in their direct vicinity cannot be resolved properly by the PIV method. In particular, an artificial spatial broadening of the shock region is produced, due to particle inertia and finite spatial and temporal resolution (window size and pulse separation). In addition, the measured velocity field is further distorted as a result of optical refraction effects when imaging the particles through the density interface of the shock wave (Elsinga et al. 2005a, b). Any unsteadiness of the shock may further add to the apparent broadening of the shock region in the measured mean velocity field.

This combination of effects may put the feasibility of pressure-gradient integration across a shock (region) under question. The consequence of including the shock region in the integration, as well as the potential performance under such circumstances is investigated in this section in more detail, using an analytical approach, a simulated experiment as well as experimental data for an oblique shock wave.

\subsection{Analytical study}

The characteristics of the pressure-gradient integration across an apparent shock region is first investigated analytically, by modelling the flow as steady and onedimensional (normal shock). The velocity field is given as a one-dimensional (uni-directional) velocity field $(u, v)=$ $(u(x), 0)$, where the (normal) velocity component $u(x)$ near the shock location may be affected by the distorting effects mentioned above (particle delay, finite spatial resolution, optical distortion effects, etc.), but is assumed to correspond to the correct physical values at a sufficient distance upstream and downstream of the shock. For the onedimensional flow description, the temperature relation from Eq. (4) is simplified, with $V=u$, as:

$$
\frac{T}{T_{\infty}}=1+\frac{\gamma-1}{2} M_{\infty}^{2}\left(1-\frac{u^{2}}{u_{\infty}^{2}}\right)
$$

Assuming further that $u(x)$ is a continuous function, the relations governing the pressure integration, Eqs. (3)-(4), can be solved analytically. This allows the pressure to be expressed directly in terms of the velocity, but different results apply for the non-conservative and conservative formulations, respectively:

$$
\frac{p}{p_{\infty}}=\frac{1+u_{\infty}^{2} / R T_{\infty}}{1+u^{2} / R T}=\frac{1+\gamma M_{\infty}^{2}}{1+\gamma M_{\infty}^{2}\left(u^{2} / u_{\infty}^{2}\right) /\left(T / T_{\infty}\right)^{2}}
$$

$$
\frac{p}{p_{\infty}}=\left(\frac{T}{T_{\infty}}\right)^{\frac{\gamma}{\gamma-1}}=\left(1+\frac{\gamma-1}{2} M_{\infty}^{2}\left(1-\frac{u^{2}}{u_{\infty}^{2}}\right)\right)^{\frac{\gamma}{\gamma-1}}
$$

(non-conservative formulation)

A first important observation resulting from this analysis is that in both cases the local pressure (ratio) is directly related to the local velocity (ratio), being further dependent only on the upstream Mach number. This implies that the 
actual shape of the velocity spatial distribution, $u(x)$, does not affect the pressure value computation in terms of $p(u)$. Hence, theoretically, the value of the pressure obtained downstream of the shock is independent of the amount of particle delay and other local distorting effects, and depends only on the particular pressure-integration strategy. Evidently, these effects do affect the pressure computation inside the shock region, where the computed pressure deviates from the true physical pressure anyway. The two pressure-velocity relations have been plotted in Fig. 3, for an upstream Mach number of 1.4. The circles indicate the pre- and post-shock conditions according to shock theory (velocity ratio $=0.592$, pressure ratio $=$ 2.120).

The result of using the conservative formulation of the momentum equation (15), is seen to return a pressure rise over the shock that is in agreement with shock theory. This correct behaviour can be understood, as the momentum equation in conservative form holds over the shock, irrespective of the internal structure of the shock region itself (Anderson 2003). On the other hand, the pressure-integration scheme using the non-conservative formulation of the momentum equation is seen from Eq. (16). to return the isentropic pressure (as predicted in Sect. 3.2), meaning that the total pressure change over the shock is not reproduced. As a consequence of this, it returns a higher pressure value after the shock (pressure ratio $=2.213$, so a $4.4 \%$ higher pressure value in this case).

For this configuration it is rather straightforward to extend the analysis to alternative integration schemes, involving a different selection of the governing equations. Here the following alternative pressure computation strategies are considered:

1. use of continuity equation, momentum equation and energy equation (adiabatic condition). As the continuity equation is invoked, there is no distinction between

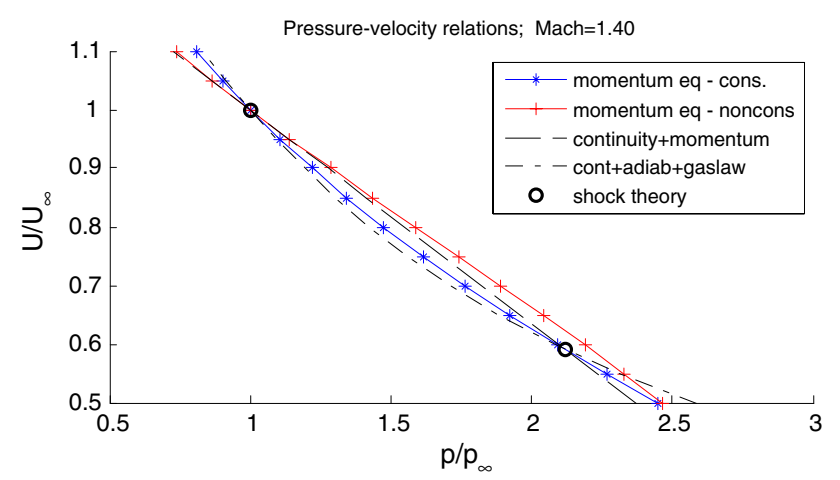

Fig. 3 Comparison of pressure-velocity relations for different flow models and exact shock wave theory (circles); computations apply to a normal shock with $M_{n}=1.40$ the conservative or non-conservative formulation of the momentum equation. The resulting pressurevelocity relation is:

$\frac{p}{p_{\infty}}=1+\frac{\gamma-1}{2} M_{\infty}^{2}\left(1-\frac{u}{u_{\infty}}\right)$

(continuity + momentum)

2. use of continuity equation, momentum equation and gas law. This strategy only differs from $(i)$ by the way in which the temperature is computed, but the pressure-velocity relation is the same as above.

3. use of continuity equation, energy equation (adiabatic condition) and gas law. Note that in this case the momentum equation is not used. The resulting pressure-velocity relation for this strategy is:

$\frac{p}{p_{\infty}}=\frac{u_{\infty}}{u}\left(1+\frac{\gamma-1}{2} M_{\infty}^{2}\left(1-\frac{u^{2}}{u_{\infty}^{2}}\right)\right)$

(continuity + adiabatic + gas law)

The two alternative pressure-velocity relations have been plotted in Fig. 3 (the black dashed lines) from which it can be observed that also these two relations reproduce the correct pressure ratio over the shock provided the correct velocity ratio as input, and hence they also produce the correct total pressure after the shock.

An important inference from this analysis is that different theoretical models, although they are theoretically equivalent under the assumption of inviscid flow, produce different results when applied as a post-processing scheme to a given velocity field, in case the velocity field departs from an inviscid flow solution.

The primary point of attention here is the comparison of the two schemes developed in the previous sections and to assess how they are capable of reproducing the correct conditions after the shock. The analytical study reveals that indeed the conservative and non-conservative schemes perform differently in the integration over a shock region, as already suggested in Sect. 3.2. The conservative scheme is in this sense to be naturally preferred, as it is predicted to return the correct pressure over the shock. The non-conservative formulation, on the other hand, introduces a systematic error in the pressure computation, which corresponds to transporting the pressure according to isentropic relations, which is incorrect. Also this finding agrees with Sect. 3.2. (More extensive comparisons of the two schemes that support these conclusions can be found in Van Oudheusden and Souverein 2007).

From this point onwards in this study the non-conservative pressure integration approach will therefore be abandoned in favour of the conservative pressure integration approach, for all subsequent further analysis and computations. 
4.2 Simulated experiment and the effect of spatial resolution

To assess the effects on the pressure computation of representing the velocity field on a finite spatial grid and of numerical truncation errors in the computation, a numerical 1D experiment was performed. The velocity field $u(x)$ is generated assuming an exponential relaxation downstream of the theoretical shock position:

$\frac{u-u_{2}}{u_{\infty}-u_{2}}=\exp \left(-x / l_{\text {relax }}\right) ; \quad x \geq 0$

where $u_{2}$ is the velocity far downstream and which corresponds to the theoretical velocity behind the shock. The velocity is sampled at discrete points $x_{i}=i \cdot \Delta x$, where $i$ is an integer number and $\Delta x$ the constant grid spacing. The effect of spatial resolution was investigated by changing the grid spacing $\Delta x$ relative to the relaxation length $l_{\text {relax }}$. Figure 4 displays the results corresponding to a normal shock with upstream Mach number 1.40, for $\Delta x / l_{\text {relax }}=0.2$ (top) and 0.5 (bottom), which are values in the typical range encountered under realistic experimental conditions (depending on particle lag and spatial resolution). The numerical results (symbols) are observed to agree well with the analytical predictions (lines), regarding the change of static and total pressure with spatial distance. Deviations between computations and theory are due to numerical
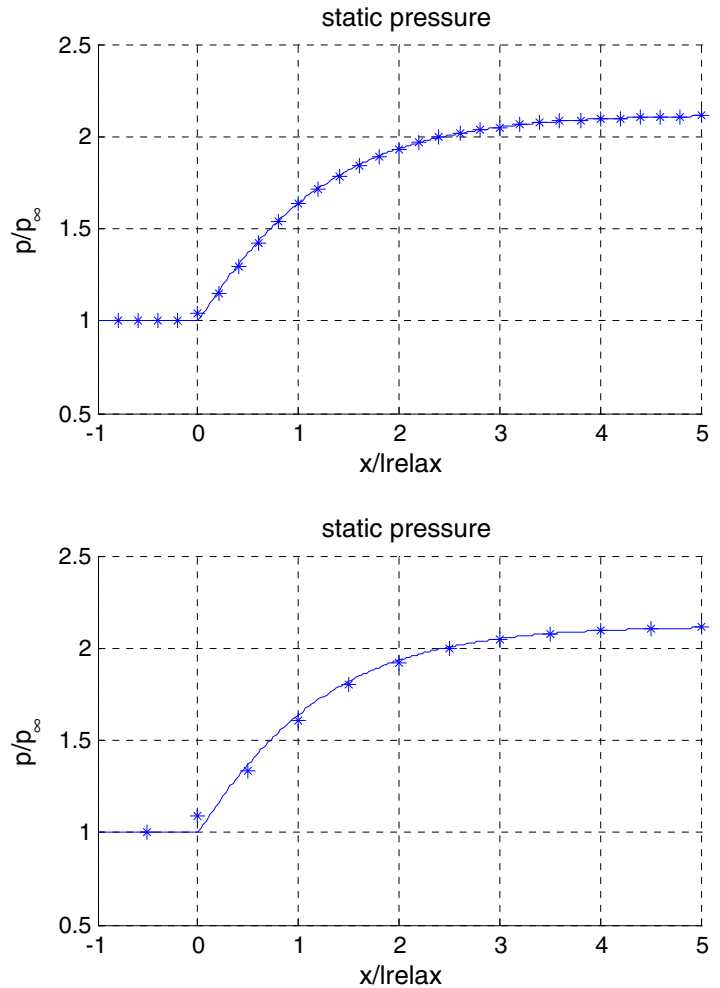

truncation errors. As mentioned, with the use of a secondorder numerical scheme, errors are expected to be proportional to velocity second-order spatial derivatives and dimensional analysis accordingly suggests that the pressure error is quadratic in the spatial grid step size $\Delta \mathrm{x}$ :

$\frac{\Delta p}{p_{\infty}} \sim-\frac{(\Delta x)^{2}}{u_{\infty}} \frac{\mathrm{d}^{2} u}{\mathrm{~d} x^{2}}$

The computed results agree with this behaviour for the continuous part of the velocity curve. A different error behaviour is observed for the specific point at the shock position $(x=0)$, due to the slope discontinuity in the analytical curve. This causes the numerical estimate of the local curvature to steepen with decreasing step size, in proportion to $\Delta x^{-1}$, so that the local error is now linear in step size: $\Delta p \sim \Delta x$, which agrees with the observed behaviour when comparing the results of the two different step sizes. The numerical simulation moreover, indicates that the truncation errors do not accumulate over the integration distance, hence, the effect of discrete sampling and numerical integration does not appear to be prohibitive in the pressure determination.

\subsection{Experimental shock test}

An experimental assessment of the pressure-gradient integration over a shock was performed using a spatial subset
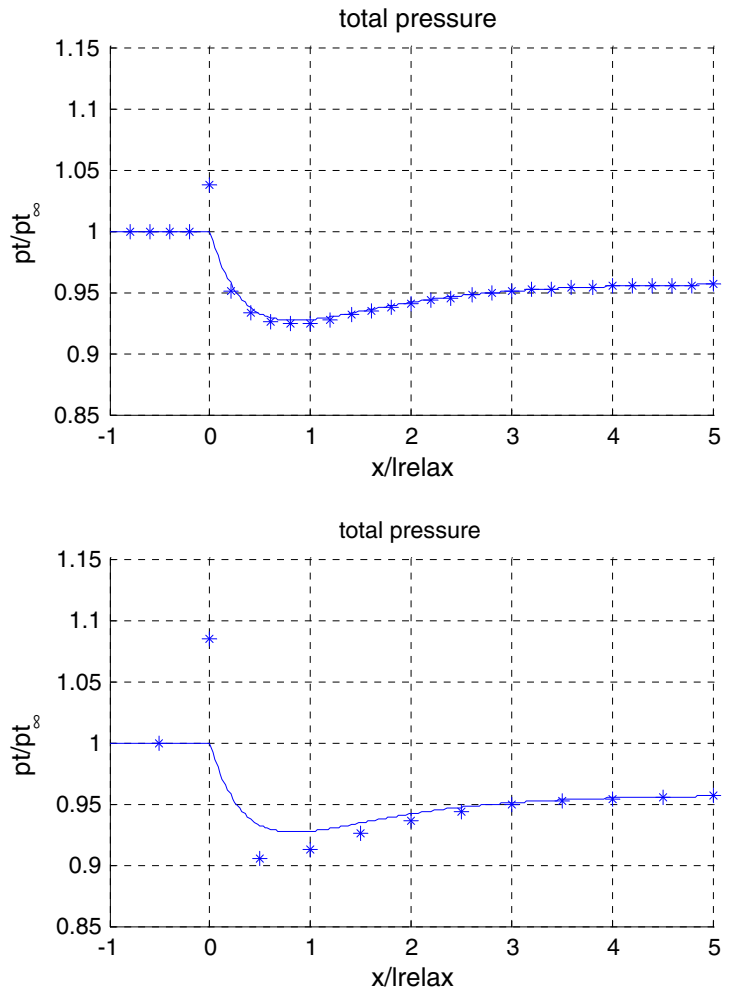

Fig. 4 Pressure integration across a velocity relaxation region corresponding to a normal shock with $M_{n}=1.40$; lines indicates analytical solution, symbols numerical simulation for a sampled velocity field with $\Delta x / l_{\text {relax }}=0.2$ (top) and 0.5 (bottom) 
of PIV data obtained in a SWBLI interaction investigation (Humble et al. 2006). This constitutes a data set that is not intentionally optimized for the shock wave in respect of viewing direction and magnification, but one that is representative of an experiment where shock waves occur as part of a larger flow region, see also Sect. 5.2. At the same time, the image quality and large ensemble size $(1,500)$ ensure that the pressure integration is not strongly affected by poor data quality or statistical convergence uncertainties in the PIV data.

The experiments were performed at a freestream Mach number of $M_{\infty}=2.07\left(U_{\infty}=520 \mathrm{~m} / \mathrm{s}\right)$ and the oblique shock wave was generated by a full-span wedge imposing a nominal flow deflection of $8^{\circ}$. The flow was seeded with titanium dioxide $\left(\mathrm{TiO}_{2}\right)$ solid particles of submicron size (estimated effective particle size is about $0.5 \mu \mathrm{m}$ ). Earlier investigations showed that for this type of seeding the particle response time is in the range of 2-3 $\mu$ s (Scarano and Van Oudheusden 2003; Schrijer et al. 2006), yielding under the present conditions a relaxation length of about 0.6-1.0 $\mathrm{mm}$. The particle images were recorded with a PCO Sensicam QE at a digital resolution of $90 \mu \mathrm{m} /$ pixel. A laser pulse separation of $2 \mu \mathrm{s}$ was applied, producing particle displacements of approximately $1 \mathrm{~mm}$ (corresponding to 11 pixels) in the freestream flow. The images were interrogated using a window-deformation iterative multi-grid scheme, at $21 \times 17$ pixels window size and an overlap factor of $75 \%$. This resulted in a measurement grid resolution of 0.47 and $0.37 \mathrm{~mm}$ in the $x$ and $y$ directions, respectively.

For the shock wave test a spatial region of $90 \times 20$ vectors (42 mm wide $\times 7 \mathrm{~mm}$ high) was extracted out of the complete flow region investigated, containing the oblique shock wave. Results for the pressure-gradient integration are given in Fig. 5. As boundary conditions the pressure was prescribed by applying the isentropic condition in a small upstream domain.

Based on the measured velocity field (see Table 1), and taking an uncertainty of $\pm 1 \mathrm{~m} / \mathrm{s}$ on each velocity component into account, the actual flow deflection angle is determined to be $7.74^{\circ} \pm 0.25^{\circ}$, which corresponds to a
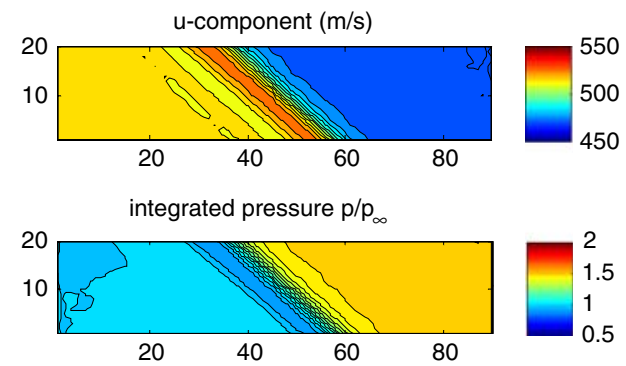

Fig. 5 Pressure integration applied to experimental PIV data for an oblique shock wave $\left(\right.$ Mach number $=2.07$, deflection angle $\left.8^{\circ}\right)$. Top velocity components (values in $\mathrm{m} / \mathrm{s}$ ); bottom static and total pressure
Table 1 Comparison of pressure-integration results for the experimental shock test

\begin{tabular}{|c|c|c|}
\hline & $\begin{array}{l}\text { Pre-shock values } \\
(s<-7 \mathrm{~mm})\end{array}$ & $\begin{array}{l}\text { Post-shock values } \\
(s>7 \mathrm{~mm})\end{array}$ \\
\hline \multicolumn{3}{|c|}{ Velocity components (mean values) } \\
\hline U-component & $518 \mathrm{~m} / \mathrm{s}$ & $471 \mathrm{~m} / \mathrm{s}$ \\
\hline V-component & $1 \mathrm{~m} / \mathrm{s}$ & $-63 \mathrm{~m} / \mathrm{s}$ \\
\hline Flow angle & $0.12^{\circ}$ & $-7.62^{\circ}$ \\
\hline Un-component $^{\mathrm{a}}$ & $304 \mathrm{~m} / \mathrm{s}$ & $224 \mathrm{~m} / \mathrm{s}$ \\
\hline Ut-component $^{\mathrm{a}}$ & $419 \mathrm{~m} / \mathrm{s}$ & $419 \mathrm{~m} / \mathrm{s}$ \\
\hline \multicolumn{3}{|c|}{ Velocity magnitude ratio } \\
\hline Experiment & 1 & 0.917 \\
\hline Theory $^{\mathrm{a}}$ & 1 & $0.920 \pm 0.003$ \\
\hline \multicolumn{3}{|l|}{ Un ratio } \\
\hline Experiment & 1 & 0.738 \\
\hline Theory $^{\mathrm{a}}$ & 1 & $0.739 \pm 0.007$ \\
\hline \multicolumn{3}{|l|}{ Temperature ratio } \\
\hline Experiment & 1 & 1.137 \\
\hline Theory $^{\mathrm{a}}$ & 1 & $1.132 \pm 0.005$ \\
\hline \multicolumn{3}{|l|}{ Pressure ratio } \\
\hline Theory $^{\mathrm{a}}$ & 1 & $1.533 \pm 0.020$ \\
\hline Pressure integration & 1.002 & 1.538 \\
\hline Isentropic & 1.001 & 1.566 \\
\hline \multicolumn{3}{|l|}{ Total pressure ratio } \\
\hline Theory $^{\mathrm{a}}$ & 1 & $0.992 \pm 0.001$ \\
\hline Pressure integration & 1.001 & 0.982 \\
\hline
\end{tabular}

${ }^{\text {a }}$ Indicate values according to exact shock theory, computed for a shock angle of $35.67^{\circ} \pm 0.25^{\circ}$

shock-normal Mach number of $M_{n}=1.207 \pm 0.007$ and a shock wave angle of $35.67^{\circ} \pm 0.25^{\circ}$. Based on the velocity magnitude ratio over the shock on the other hand, which is $0.9170 \pm 0.0039$, a corresponding deflection angle of $7.96^{\circ}$ $\pm 0.35^{\circ}$ is inferred (shock wave angle of $35.88^{\circ} \pm 0.35^{\circ}$, $\left.M_{n}=1.213 \pm 0.010\right)$. The nominal values of the two methods are in statistical agreement in view of the uncertainty intervals. The values obtained with the first method (mean and uncertainty range) have been used in a further

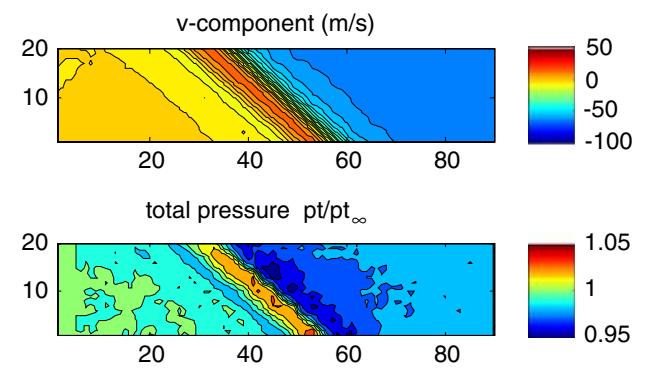

scaled with free stream values. Indices refer to row and column number of the vector data grid 
comparison of the experimental results to the 1D shockregion analysis of Sect. 4.1. Along the central horizontal line in the field of view, the velocity components normal and tangential to the shock have been computed and have been plotted in Fig. 6 (top) against the distance measured normal to the shock wave. The origin $s=0$ is set at the estimated actual shock location. The bottom diagrams display the pressure profiles (static and total) along that same line, obtained with the pressure-integration scheme. The results of the 1D analysis, see Eq. (15), using the shock-normal velocity component in combination with the temperature based on the complete velocity information, have been added in red for comparison, as well as the pressure prediction from isentropic theory (black dashed line), see Eq. (1). In the velocity and derived pressure fields some artifacts are revealed that are associated with the inherent limitations of the measurement technique, in particular the spatial broadening of the shock region due to particle inertia and finite window size and pulse delay time. Also, upstream distortions over a distance of about $6 \mathrm{~mm}$ are observed, that can be attributed to optical refraction effects due to the imaging of the particles through the
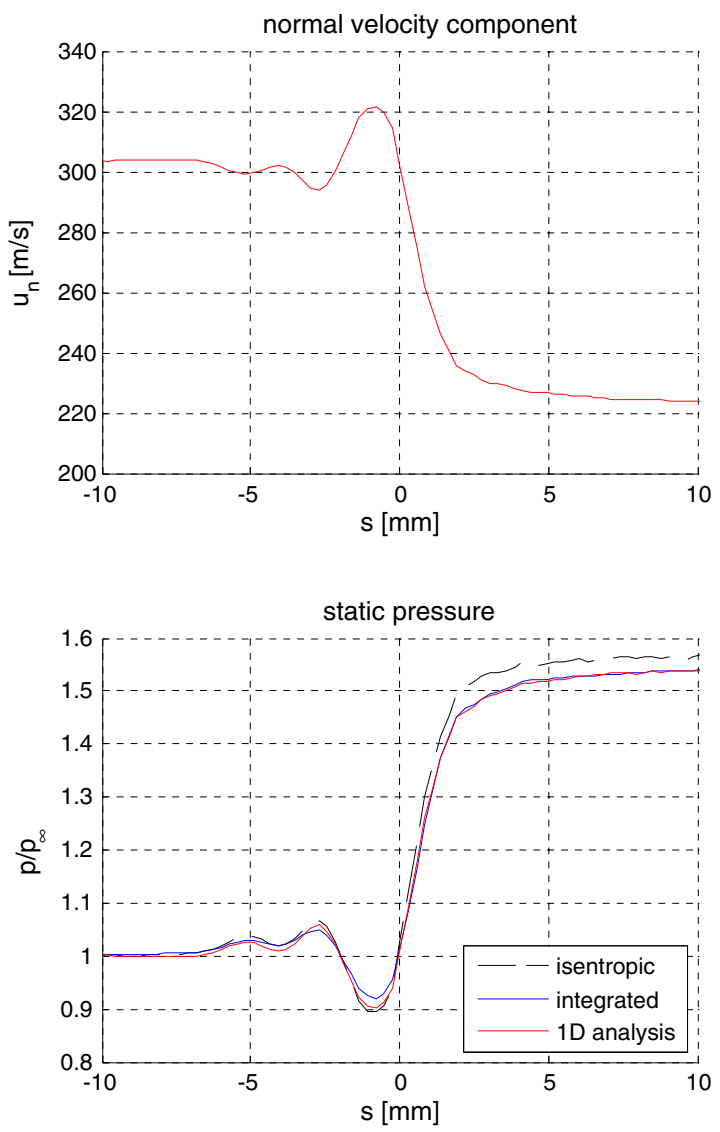

Fig. 6 Velocity and pressure profiles along a horizontal line in the center of the field of view (pixel row $=10$ ); $s$ is the distance measured perpendicular to the shock. Top velocity components normal and density interface of the shock wave; a proper adjustment of the viewing angle can reduce these effects significantly (Elsinga et al. 2005a, b).

The values in Table 1 indicate that the pressure-integration scheme returns a pressure after the shock that is in good agreement with shock theory (difference with respect to the nominal theoretical value is $0.3 \%$ and well within its uncertainty). The variation with spatial distance $s$ of the total pressure as derived from the integrated pressure, see Fig. 6 (bottom-right), displays with respect to the results of the 1D analysis the additional effect of numerical truncation error, similarly as in Fig. 4. The decrease in total pressure over the shock, of about $2 \%$, is larger than that according to shock theory (which is about $1 \%$ ), see Table 1 . As this value is reproduced also by the $1 \mathrm{D}$ analysis (red curve) it is not an artefact of the spatial integration, but is related to the underlying velocity information. From the values in Table 1, it can be seen that the cause lies in the slight overprediction of the temperature ratio over the shock w.r.t. theory, which is a consequence of the smaller value of the velocity magnitude ratio. Hence, it can be concluded that the slight inconsistencies in the velocity field information
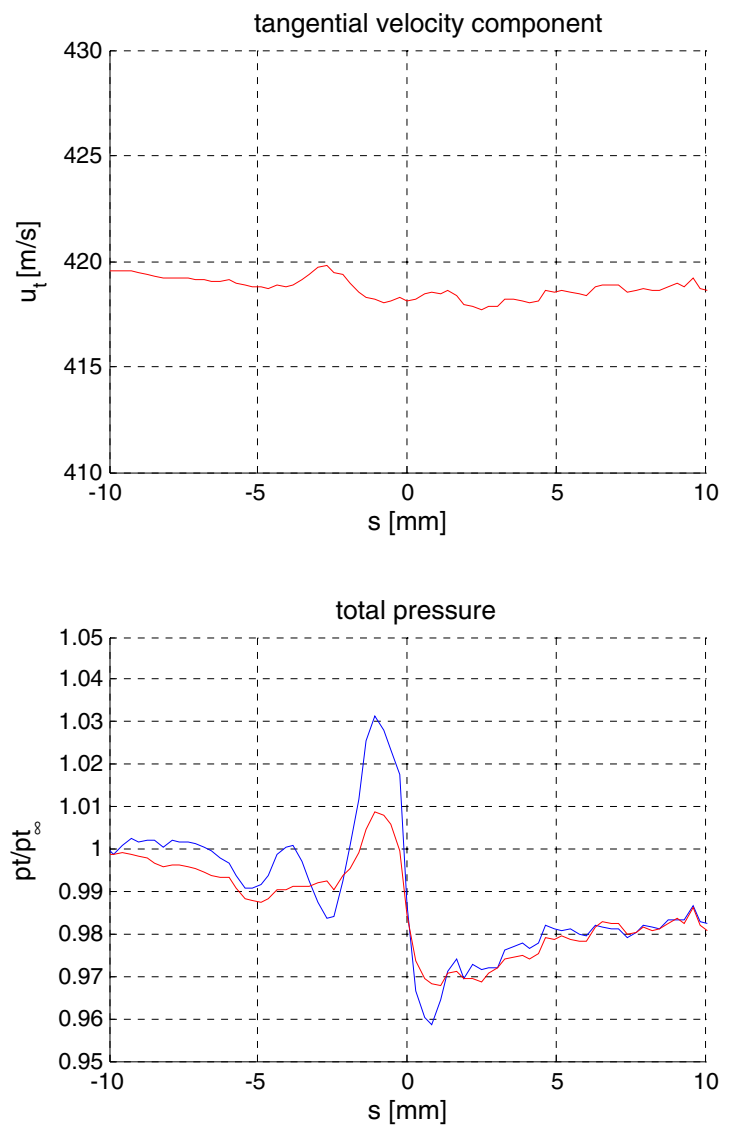

parallel to the shock, bottom static and total pressure for the integration procedure and comparison to isentropic flow results (constant total pressure) and the "1D analysis" of Sect. 4.1 
with respect to exact theory (recall the discussion on the shock wave angle determination at the beginning of this section) introduces an uncertainty on the pressure computation that directly affects the total pressure as well.

\subsection{Conclusions of the shock-region assessment}

The particular objective of the current section has been to assess the feasibility to propagate pressure information over a shock wave region by means of the proposed integration procedure, as well as investigating the possible influence of the formulation in which the momentum equation is used (conservative or non-conservative). Firstly, although within the apparent and artificially broadened shock region clearly the pressure computation does not yield correct values for the region itself, the pressure integration across a shock region appears to be feasible when the conservative formulation of the momentum equation is implemented.

The analytical study of the shock region in Sect. 4.1 illustrates that different combinations and/or formulations of the governing equations, although theoretically equivalent within the inviscid flow model, produce different pressure-velocity relations. According to this analysis the conservative formulation returns for the pressure downstream of the shock the correct value, i.e. one that is in agreement with shock theory (provided that the correct velocity ratio is applied), whereas the non-conservative formulation introduces a bias error by returning the isentropic pressure. The latter observation is in agreement with the analysis of Sect. 3.2. For this reason, the conservative formulation has been adopted for further use in this study.

The numerical simulation indicates that for typical experimental settings the effect of discrete sampling and numerical integration is not prohibitive to the pressure determination. The experimental shock test further reveals that small inconsistencies in the velocity data set can affect the pressure computation across the shock, which may introduce additional errors on the pressure, that were found to be of the order of $1 \%$ for the experimental data considered.

\section{Application to experimental data sets}

\subsection{Bi-convex airfoil in supersonic flow}

As a first complete experimental test case for the computation of pressure in a compressible flow, the situation of a 2D airfoil in a supersonic freestream flow is considered (Souverein et al. 2007). Experiments were performed in the TST-27 blow-down transonic-supersonic wind tunnel (see Fig. 7); test section dimensions are $280 \mathrm{~mm} \times 255 \mathrm{~mm}$ (width $\times$ height). Tests were carried out on a full span biconvex airfoil with a chord of $100 \mathrm{~mm}$ and a thickness of $12 \mathrm{~mm}$. For the experimental conditions considered here, the incidence angle was $0^{\circ}$ and the freestream Mach number 1.96 (freestream velocity is $494 \mathrm{~m} / \mathrm{s}$ ). The stagnation pressure was $196 \mathrm{kPa}$, for which the corresponding Reynolds number is $2.7 \times 10^{6}$ based on the model chord.

The flow was seeded with submicron titanium dioxide particles (see Sect. 4.3) which were dispersed in the settling chamber of the wind tunnel. Illumination was provided by a Big Sky Laser CFR PIV-200 Nd:YAG laser, producing a $1.5 \mathrm{~mm}$ thick light sheet. The laser wave length is $532 \mathrm{~nm}$ and the energy is $200 \mathrm{~mJ} /$ pulse, with a pulse duration of 6 ns. A field of view of $146.5 \times 80.1 \mathrm{~mm}^{2}$ was imaged with a $1,280 \times 1,024$ (vertical image size cropped to 800) pixel CCD camera. With illumination from behind, a small shadow region upstream of the model occurs, which is excluded from the pressure integration. A pulse separation of $3 \mu$ s was applied, yielding a maximum particle displacement of 15 pixels. Image analysis was carried out with the same software as described before. A window size of $31 \times 31$ pixels was applied with an overlap of $75 \%$, yielding a measurement grid with uniform spacing of about $0.9 \mathrm{~mm}(0.9 \%$ chord $)$. A data ensemble size of about 200 image pairs was obtained at an acquisition rate of $4.2 \mathrm{~Hz}$.

The diagrams in Fig. 8 (top row) depict the velocity components, normalized with the free stream velocity. The model region has been masked (slightly extended in view of perspective), as is the edge of the observation window, while the upstream shadow region was patched with the uniform value of the upstream velocity. The pressure
Fig. 7 Experimental setup for supersonic biconvex airfoil investigation. Left mounting of the airfoil model, right schlieren visualization (flow direction is from left to right)
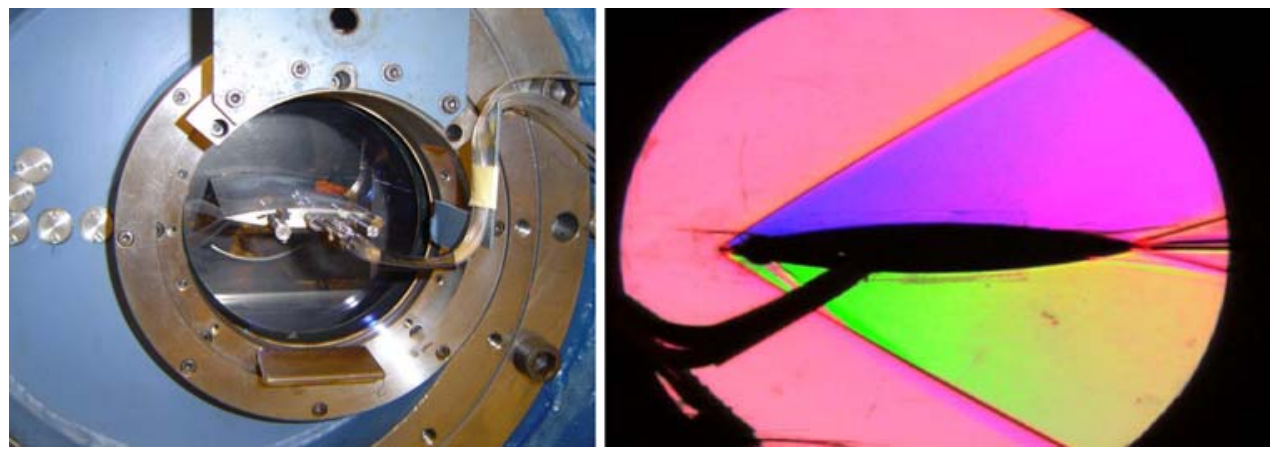

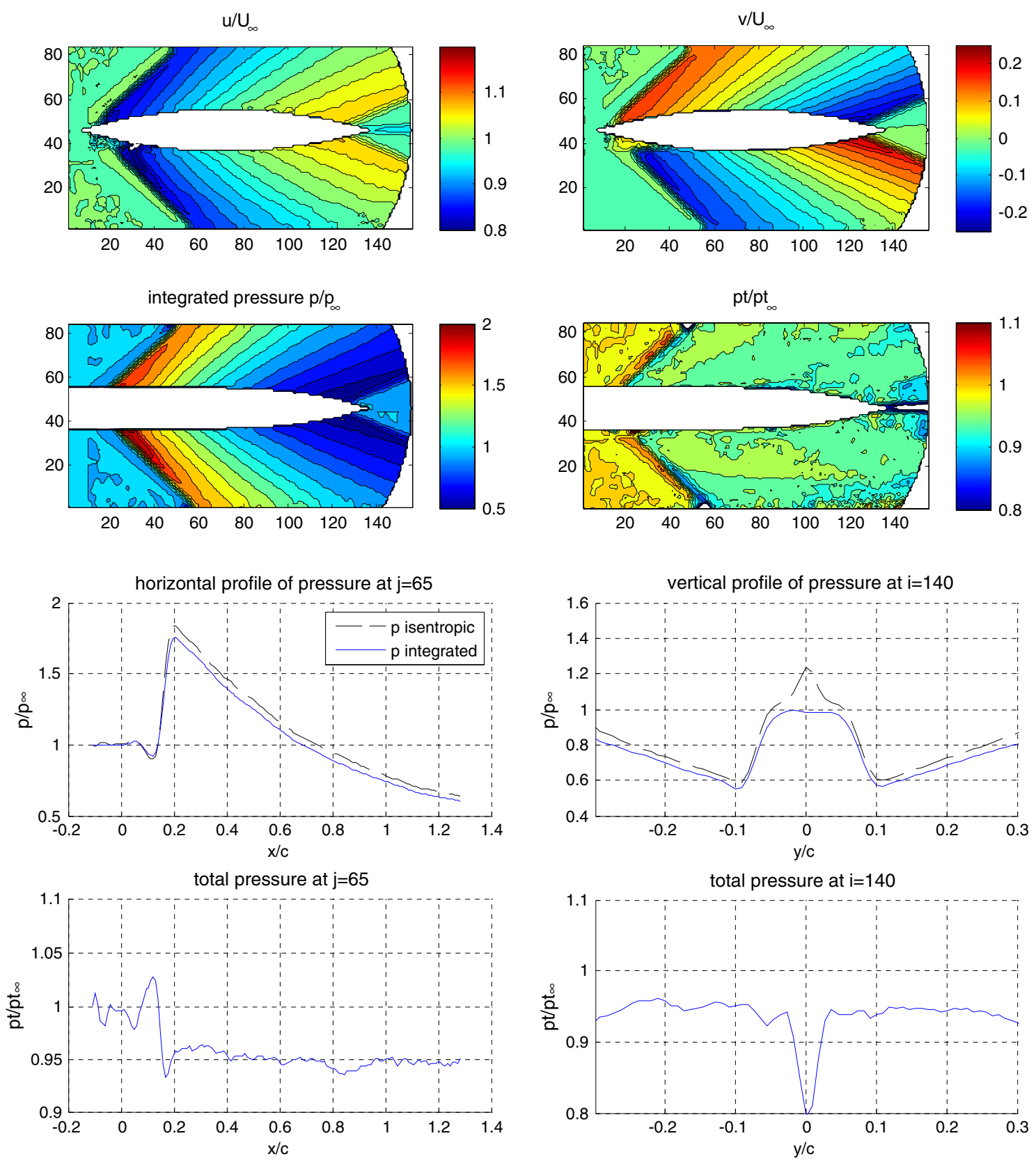

Fig. 8 Results of pressure integration applied to the flow around a biconvex airfoil (Mach $=1.96$ ). Top velocity and pressure fields, bottom static and total pressure profiles along a horizontal line above the model $(y / c=0.17)$ and along a vertical line across the wake $(x / c=1.16)$

integration was performed with uniform pressure imposed in a strip upstream of the airfoil. The shadow region directly upstream of the model was excluded from the integration. The integration was first performed in two horizontal bands above and below the model, and subsequently extended towards the model and into the wake, in order to prevent that measurement artefacts close to the model surface affect the pressure integration in the external flow away from the model. The static and total pressure fields (scaled with freestream values) that were obtained with the pressure-integration method have been depicted in the second row of the Figure. The remaining diagrams present static and total pressure profiles along selected lines across the flow field (one horizontal at $y / c=0.17$ above the model, one vertical at $x / c=1.16$ crossing the wake behind the model). The isentropic pressure has been included for comparison. Clearly, the integrated pressure returns a lower pressure than the isentropic behind the bow shock, with a total-pressure drop of about $4-5 \%$. This value is slightly in excess of what shock-wave theory would predict. In view of the impinging expansion waves originating from the curved airfoil surface, the bow-shock is not of 
Fig. 9 Experimental

configuration of the SWBLI experiments; left view of the test section with side-wall mounted shock generator; right schlieren visualization of the interaction
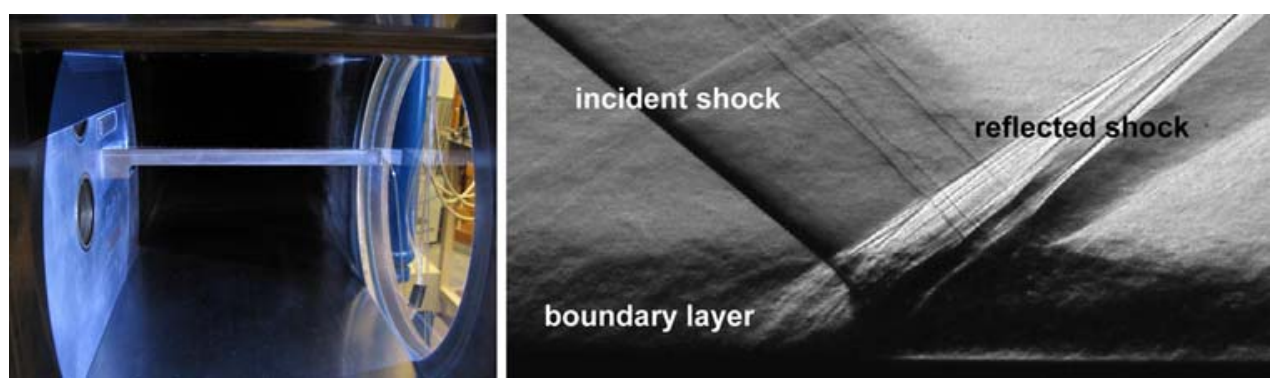

constant strength. Its maximum strength occurs at the leading edge, with a theoretical flow deflection of $13.7^{\circ}$. For this, shock-wave theory predicts a static and total pressure ratio of 2.040 and 0.964 , respectively (shock Mach number $=1.375$ ). Note also that the unphysical pressure recovery of the isentropic theory in the wake is removed by the pressure integration method, which basically maintains a constant pressure across the wake.

\subsection{Shock wave boundary layer interaction}

The SWBLI experiments were performed in the same wind tunnel as described in Sect. 5.1, run at a freestream Mach number of $M_{\infty}=2.07\left(U_{\infty}=520 \mathrm{~m} / \mathrm{s}\right)$, stagnation pressure of $280 \mathrm{kPa}$ and stagnation temperature of $284 \mathrm{~K}$. The oblique shock wave was generated by a full-span wedge imposing a nominal flow deflection of $8^{\circ}$ (Fig. 9, left). The interaction configuration studied was that of the shock impinging on the turbulent boundary layer on the test section wall (Fig. 9, right). The boundary layer has a thickness $\delta$ of approximately $20 \mathrm{~mm}$ and was assessed to be in fully developed turbulent condition, with the Reynolds number based on momentum thickness equal to 49,000. Full details of the experimental investigation can be found in Humble et al. (2006).

Flow seeding was the same as described in previous sections. Illumination was provided by a Spectra-Physics Quanta Ray double-pulsed Nd:Yag laser with $400 \mathrm{~mJ} /$ pulse energy and a $6 \mathrm{~ns}$ pulse duration, at a wavelength of 532 $\mathrm{nm}$. The light sheet thickness was approximately $1.5 \mathrm{~mm}$. The particle images were recorded with a PCO Sensicam QE (12-bit, 1,376 × 1,040 pixel). Only 432 pixels were used in the vertical direction given the aspect ratio of the interaction region of interest and in addition permitting an increased image recording rate of $10 \mathrm{~Hz}$ to be obtained. The flow was imaged over a FOV of $124 \times 39 \mathrm{~mm}$ (approx. $6 \delta \times 2 \delta$ ) at a digital resolution of $90 \mu \mathrm{m} /$ pixel. The details of the PIV recording parameters and imageinterrogation settings have been given in Sect. 3.2.

The spatial distributions of the velocity components $u$ and $v$, as well as the results of the pressure integration are given in Fig. 10, where all velocities were scaled with
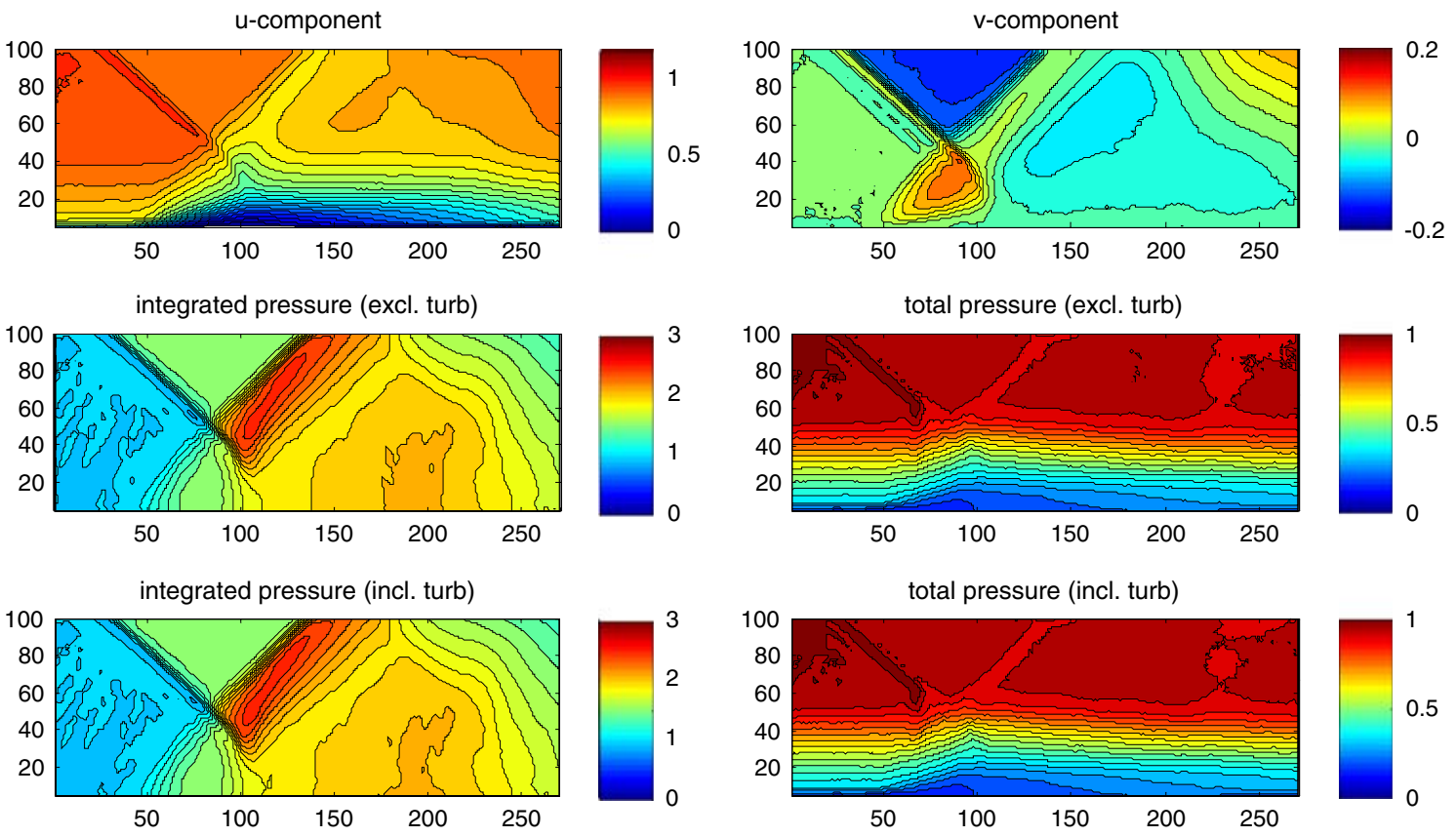

Fig. 10 Pressure-integration results for the SWBLI experiment-velocity and pressure fields 
freestream velocity and static and total pressures with their respective freestream values. The integration was initiated with isentropic pressure values in the top-left corner, over a region extending over 20 vectors high by 20 vectors wide. First a horizontal strip of 40 vectors height was integrated over the entire streamwise extent, yielding an external flow pressure region from which the pressure integration was subsequently progressed downwards. The pressure integration results are given for two cases, viz., with or without the turbulence terms included. The graphs in Fig. 11 provide pressure profiles at selected locations in the flow. The first gives static and total pressure in the external flow well outside the boundary layer $(y=32 \mathrm{~mm}=1.6 \delta)$, the second the static pressure near the wall (at $y=1.1 \mathrm{~mm}$ ). Appreciable total pressure changes occur over the shocks (especially the second); according to shock-wave theory the decrease in total pressure should be about $1 \%$ for each shock, the pressure-gradient integration with the conservative scheme yields a total decrease of about $5 \%$.

The effect of including the turbulence terms is relatively small, and it is difficult to assess its magnitude based on the direct comparison of the two pressure fields. Therefore, the differences in static and total pressure between the different computations have been separately plotted in Fig. 12 (top row) which shows that the effect is less than $5 \%$ of the reference pressure. The contributions of the individual turbulence components have been assessed, by repeating the pressure integration, each time with only one of the
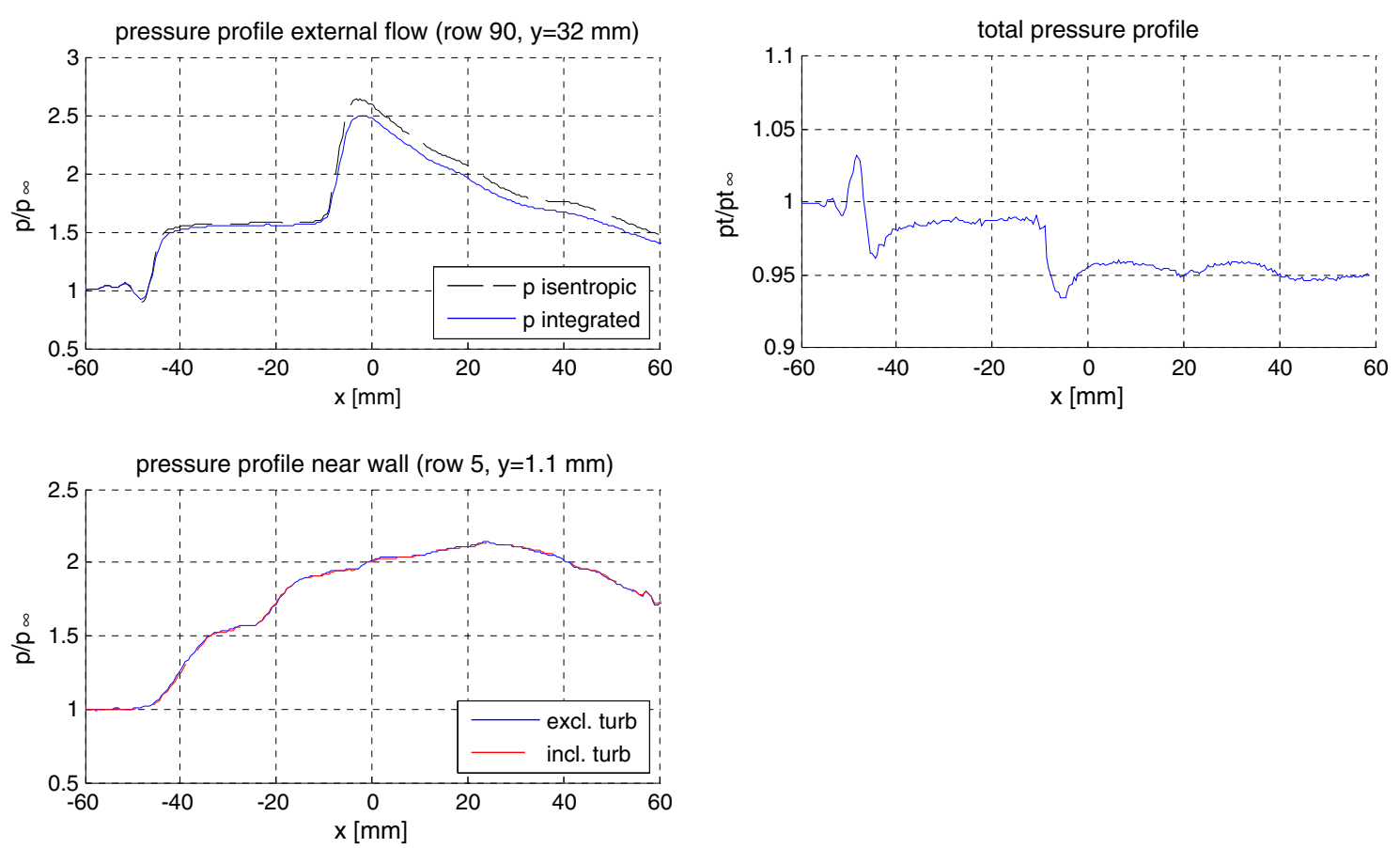

turbulence components enabled in the computation (subsequent rows). It is observed that, notwithstanding that the $\mathrm{u}$-component is the largest in magnitude, it has a negligible influence on the pressure field, while a more prominent effect is observed from the transverse component (especially in the boundary layer downstream of the interaction) and the Reynolds shear stress (especially in the immediate shock-interaction region inside the boundary layer). Validation of the turbulence data (Humble et al. 2006) has shown that normal stresses are measured correctly (with uncertainty of ca. 5-10\%) until close to the wall (down to ca $0.1 \delta$ ) while the Reynolds stress is correct (uncertainty 10-20\%) down to ca $0.25 \delta$. For this reason, conclusions on the contribution of the turbulent terms are not governed by the error on the turbulence terms themselves.

\section{Conclusions}

The computation of (mean) pressure fields from PIV velocimetry data through application of the momentum equation requires additional flow governing equations to be invoked in the case of compressible flow, to account for the variable density. A convenient solution for this was devised by combining the gas law and the adiabatic flow assumption (energy equation), together with the momentum equation with the viscous terms removed. In the study the difference was assessed when the momentum equation is

extrapolated intersection point of the shock wave with the wall 

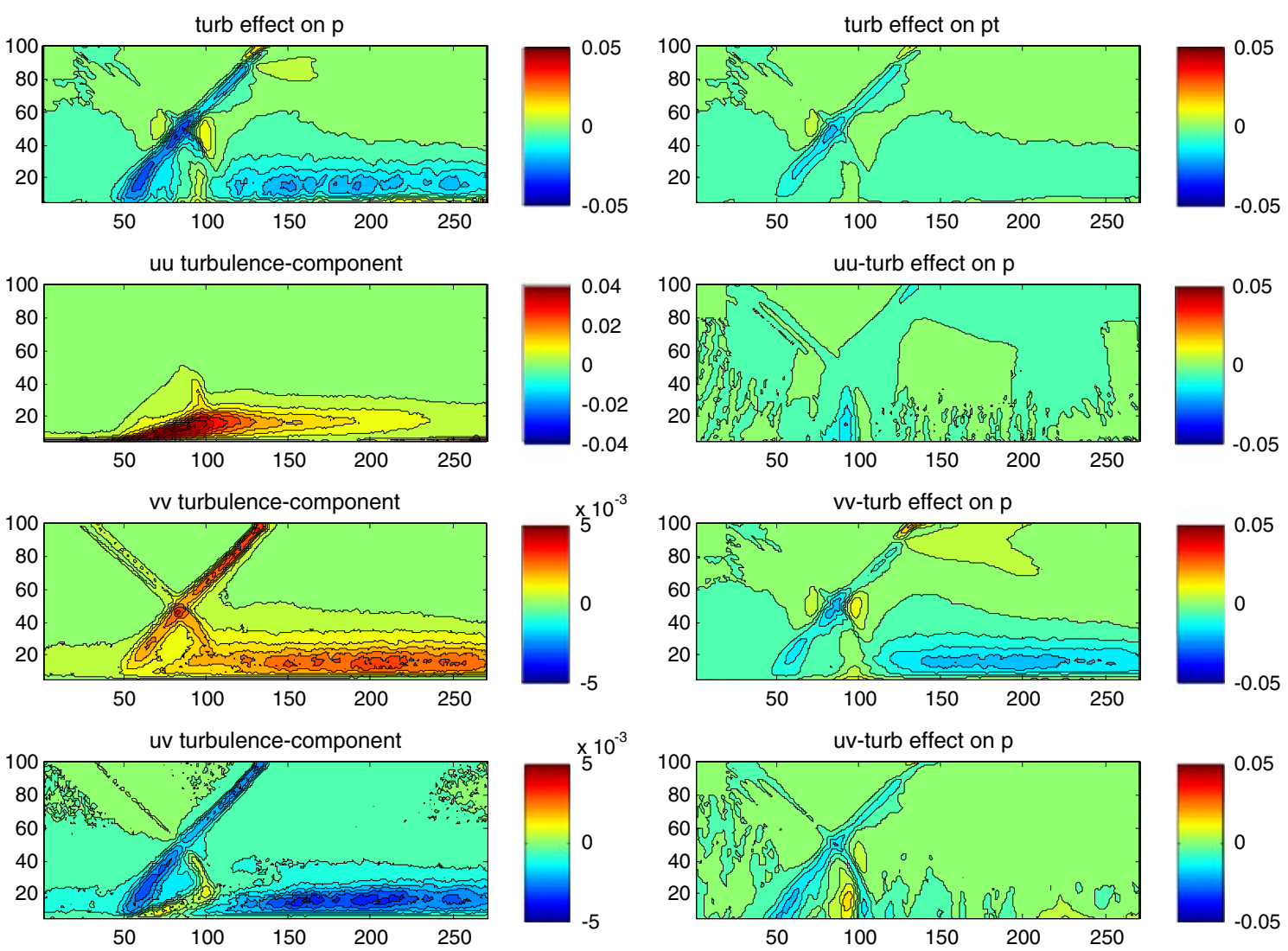

Fig. 12 Effect of turbulence terms on the pressure-integration results. Top row: effect of the turbulence terms on the computation of static and total pressure. Subsequent rows: magnitude of individual turbulence components (left) and their effect on the pressure computation (right)

used in either the conservative or non-conservative form. Although from theoretical arguments this should be equivalent in view of mass conservation, this does not guarantee that the different schemes will perform identically under all conditions, as the conservation of mass is not explicitly imposed in the procedure.

An explicit formulation for the pressure gradient problem was obtained, which allows computation of the pressure field non-iteratively, employing a spatial-marching gradient-integration scheme.

Furthermore, compressible flows display specific flow features, notably shocks but also thin shear layers that pose particular difficulties for the flow velocity measurement and the subsequent determination of the pressure field. It was shown that in principle and practice the pressure integration can be extended through shock regions, which was verified using experimental shock wave data.

The distinction between conservative and non-conservative formulation was found not to be relevant for the pressure integration in those flow regions where the flow is essentially inviscid (but possibly rotational). There is an essential difference for these two schemes, however, in the way that they propagate the pressure information across a shock region. With the conservative formulation the pressure returned is in theoretical agreement with the shock relations, whereas the non-conservative formulation returns the isentropic pressure. This leads to a natural preference for the conservative formulation, which was the pressureintegration strategy subsequently used for all further computations.

The experimental shock test further revealed that small inconsistencies in the velocity data set can affect the pressure computation across the shock, which may introduce additional errors on the pressure that were found to be of the order of $1 \%$ for the experimental data considered. The pressure-integration procedure was subsequently applied to extract the pressure field of a supersonic flow around a biconvex airfoil and in a shock wave boundary layer interaction. Also in these experiments the total pressure drop over the shocks was somewhat larger than that according to theory.

The shock regions indeed appear to be the most critical regions in the pressure-integration approach. Outside these regions, as evidenced by the different experiments, the resulting pressure error remains quite small. In regions that can be considered isentropic (upstream or behind shocks), the total pressure shows variations of typically much less than $1 \%$, which indicates that effect of velocity uncertainty 
and numerical truncation error under these conditions do not seriously hamper the pressure-integration error.

The experimental evaluation of the SWBLI data further indicated that even under these conditions of relatively high turbulence activity, the contribution of the turbulence terms to the pressure computation is small, of the order of at most $5 \%$ of the free stream pressure.

Acknowledgments The PIV velocity field data and the illustrations of the experimental setup for the biconvex airfoil and the shock-wave boundary-layer interaction were kindly provided by L.J. Souverein and R.A. Humble, respectively.

Open Access This article is distributed under the terms of the Creative Commons Attribution Noncommercial License which permits any noncommercial use, distribution, and reproduction in any medium, provided the original author(s) and source are credited.

\section{References}

Anderson JD (2003) Modern compressible flow with historical perspective, 3rd edn. McGraw-Hill, New York

Baur T, Köngeter J (1999) PIV with high temporal resolution for the determination of local pressure reductions from coherent turbulent phenomena. In: 3rd international workshop on PIV, Santa Barbara, USA, pp 671-676

Elsinga GE, Van Oudheusden BW, Scarano F (2005a) Evaluation of optical distortion effects in PIV. Exp Fluids 39:246-256

Elsinga GE, Van Oudheusden BW, Scarano F (2005b) The effect of particle image blur on the correlation map and velocity measurement in PIV. Optical Engineering and Instrumentation, SPIE Annual Meeting, 31 July-4 Aug 2005, San Diego, USA, Paper 5880-37

Fujisawa N, Tanahashi S, Srinavas K (2005) Evaluation of pressure field and fluid forces on a circular cylinder with and without rotational oscillation using velocity data from PIV measurement. Mea Sci Techn 16:989-996

Gurka R, Liberzon A, Hefetz D, Rubinstein D, Shavit U (1999) Computation of Pressure Distribution Using PIV Velocity Data. In: 3rd International workshop on PIV, Santa Barbara, USA, pp 101-106

Hosokawa S, Moriyama S, Tomiyama A, Takada N (2003) PIV measurement of pressure distributions about single bubbles. J Nuclear Sci Technol 40:754-762

Humble RA, Scarano F, Van Oudheusden BW (2006) Experimental study of an unsteady impinging shockwave/turbulent boundary layer interaction using PIV. In: 36th AIAA Fluid Dynamics Conference and Exhibit, 5-8 June 2006, San Francisco, USA, AIAA Paper 2006-3361

Liu X, Katz J (2006) Instantaneous pressure and material acceleration measurements using a four-exposure PIV system. Exp Fluids 41:227-240

Scarano F, van Oudheusden BW (2003) Planar velocity measurements of a two-dimensional compressible wake. Exp Fluids 34:430-441

Schrijer FFJ, Scarano F, van Oudheusden BW (2006) Application of PIV in a Mach 7 double-ramp flow. Exp Fluids 41:353-363

Souverein LJ, Van Oudheusden BW, Scarano F (2007) Particle image velocimetry based loads determination in supersonic flows. In: 45th AIAA aerospace sciences meeting, 8-11 Jan 2007, Reno, AIAA Paper 2007-0050

Unal MF, Lin JC, Rockwell D (1997) Force prediction by PIV imaging: a momentum based approach. J Fluids Struct 11:965-971

Van Oudheusden BW, Scarano F, Roosenboom EWM, Casimiri EWF, Souverein LJ (2007) Evaluation of integral forces and pressure fields from planar velocimetry data for incompressible and compressible flows. Exp Fluids 43:153-162

Van Oudheusden BW, Souverein LJ (2007) Evaluation of the pressure field from PIV in a shock wave boundary layer interaction, 7th International Symposium on Particle Image Velocimetry, 11-14 Sept. 2007, Rome, Italy

White FM (1991) Viscous fluid flow, 2nd edn. McGraw-Hill, New York 